\title{
Les entrevues structurées multiples pour la sélection des candidats aux programmes de formation en médecine
}

\author{
Multiple structured interviews for admission \\ into medicine training programs
}

Le présent numéro du journal présente les résultats d'une très intéressante étude menée au Québec par des chercheurs de l'Université de Sherbrooke, consacrée à l' « évaluation édumétrique d'un dispositif d'entrevues structurées multiples pour la sélection de candidats dans un programme post-gradué de dermatologie ${ }^{[1]}$. Cette technique des minientrevues multiples (MEM), inspirée des principes de l'examen clinique objectif structuré (ECOS) et de son format à stations multiples, a été développée depuis près de dix ans par des chercheurs de l'Université McMaster pour être utilisée dans le cadre de la sélection des étudiants en médecine ${ }^{[2]}$.

Traditionnellement, en Amérique du Nord, tout particulièrement au Canada, la sélection des étudiants souhaitant s'engager dans les études médicales reposait sur deux critères principaux : la réussite scolaire antérieure et la performance à une ou plusieurs entrevues individuelles et collectives. Alors que la réussite scolaire antérieure demeure le meilleur prédicteur de la réussite académique future, l'entrevue de sélection, dans sa version traditionnelle, souffre depuis fort longtemps d'un manque de fidélité et d'un pouvoir prédictif nul. Malgré ces défauts criants, les facultés de médecine canadiennes ne se résolvent pas à éliminer l'entrevue de la démarche de sélection mais ils cherchent à en contourner les défauts.

L'idée des MEM est simple et certes élégante : s'il est possible qu'un évaluateur commette une erreur de jugement lors d'une entrevue, il est moins vraisemblable que douze évaluateurs commettent tous une erreur dans le même sens. Dès lors, faire évaluer les candidats par 12 évaluateurs différents et dans 12 situations différentes devrait donner un portrait plus juste de la qualité de chacun d'entre eux ! Le choix des situations donnant lieu à l'observation et à l'évaluation est élaboré essentiellement en référence au modèle des compétences CanMeds, si cher aux Canadiens; on postule que s'il est attendu que le médecin accompli doive, in fine, démontrer la maîtrise des sept compétences prescrites, alors il est souhaitable que le candidat en médecine doive, lui aussi, déjà faire la preuve d'un soupçon significatif de ces compétences, notamment de celles relatives à des capacités non strictement «cognitives ». Ainsi, chaque station vise à mettre le candidat dans une situation exigeant de lui qu'il convainque les évaluateurs sur les plans de la communication, de la tolérance à l'ambiguïté, de l'ouverture d'esprit, de la solution de problème, bref les voltiges attendues d'un futur médecin idéal.

Sur le plan de la fidélité, les résultats obtenus par cette méthode sont, à ce jour, assez satisfaisants : selon le contexte et le nombre de stations, les coefficients de fidélité sont mesurés de 0,65 à 0,85 . Une étude récemment publiée par Eva et collaborateurs suggère que le score aux MEM est le meilleur prédicteur de la performance pour ce qui concerne les aspects non cognitifs de l'examen de certification à la fin des études médicales ${ }^{[3]}$. Cependant, la constatation de l'absence de corrélation entre les 
scores aux MEM et les scores obtenus à d'autres mesures reconnues et relatives aux dimensions dites «non cognitives » risque d'atténuer l'enthousiasme quant à la réelle valeur ajoutée que promet cette méthode ${ }^{[4,5]}$. Des données inédites, accumulées au cours des quatre dernières années dans trois universités québécoises francophones sur près de 5000 candidats, commencent à permettre d'identifier les aspects plus problématiques des MEM. Qui plus est, il apparaît que les jeunes candidats se préparent aux épreuves dans le cadre de séances d'entraînement aux MEM : difficile de ne pas y voir un risque de confusion entre le savoir-faire et le « savoir-être en vue », tant chez les candidats que chez les évaluateurs!

Cette méthode des MEM commence à inspirer les personnes responsables des procédures de sélection en vue de l'admission aux études spécialisées post-graduées ${ }^{[6,7]}$. C'est dans ce cadre que s'inscrit l'article de Bergeron et collaborateurs. Alors qu'il est difficile, peut-être même utopique, de demander à des jeunes gens de 19 ans de démontrer toute la palette de leurs habiletés sociales à l'occasion de 12 mini-situations stressantes, au cours desquelles leur avenir se joue, il est permis de penser que la méthode des MEM constitue un ajout significatif pour mettre à l'épreuve ceux et celles qui ont déjà les acquis de quatre années d'études médicales dans leurs bagages.

À l'heure du virage de l'approche par compétences, qui met l'accent sur la capacité à mobiliser et déployer des ressources dans le contexte de l'action, il n'est pas étonnant - et il convient sans doute de s'en réjouir - que l'accès aux filières de formations spécialisées puisse tenir compte de la démonstration satisfaisante de ces compétences par les futurs spécialistes en devenir. Il faut souligner aussi l'importance grandissante accordée à la simulation en tant que mode de formation et d'évaluation. À cet égard, il est intéressant de poser la question de savoir si les MEM ne seraient rien d'autre que des «erstaz» de simulation. Dans une telle perspective, alors que l'on sait développer des scénarios crédibles et valides et qu'il est possible d'élaborer avec soin des grilles d'évaluation parfaitement bien spécifiées en recourant à descripteurs qualitatifs, il est significatif de faire le constat que c'est le jugement des examinateurs qui constitue la limite principale de la procédure. Il ne s'agit pas de le regretter car, même lorsqu'elle recourt à des mesures, et comme le rappelle l'étymologie, c'est bien le jugement de valeur qui est au cœur de la démarche évaluative. L'enjeu réside alors, et c'est le sens des commentaires des auteurs, en ce que les évaluateurs consentent en revanche à se départir de leur seule impression générale implicite et à fonder leur jugement sur des critères et un processus désormais de plus en plus explicites.

\section{Robert GAGNON}

Bureau d'évaluation

Centre de pédagogie appliquée aux sciences de la santé (CPASS), Université de Montréal, Canada Mailto : robert.gagnon@umontreal.ca

\section{Références}

1. Bergeron L, St-Onge L, Martel S, Hanna D. Évaluation édumétrique d'un dispositif d'entrevues structurées multiples pour la sélection de candidats dans un programme postgradué de dermatologie. Pédagogie Médicale 2011;12:17-27.

2. Eva KW, Rosenfeld J, Reiter HI, Norman GR. An admissions OSCE: the Multiple Mini-Interview. Med Educ 2004;38:314-26.

3. Eva KW, Reiter HI, Trinh K, Wasi P, Rosenfeld J, Norman GR. Predictive validity of the multiple mini-interview for selecting medical trainees. Med Educ 2009;43:767-75

4. Kulasegaram K, Reiter HI, Wiesner W, Hackett RD, Norman GR. Non-association between Neo-5 personality tests and multiple mini-interview. Adv Health Sci Educ Theory Pract 2010;15:415-23

5. Yen W. An exploration of the relationship between emotional intelligence (EI) and the Multiple Mini-Interview (MMI). Adv Health Sci Educ Theory Pract 2011;16:59-67

6. Hofmeister M, Lockyer J, Crutcher R. The multiple mini-interview for slection of international medical graduates into family residency education. Med Educ 2009:43:573-9.

7. Dore KL, Kreuger S, Ladhani M, Rolfson D, Kurtz D, Kulasegaram K, et al. The Reliability and Acceptability of the Multiple Mini-Interview as a Selection Instrument for Postgraduate Admissions. Acad Med;85(10 Suppl): S60-3. 